\title{
The atypical cadherin flamingo determines the competence of neurons for activity- dependent fine-scale topography
}

\author{
Ruonan $\mathrm{Li}^{1,2+}$, Yuhua Liang ${ }^{1 \dagger}$, Siyang Zheng ${ }^{3}$, Qun $\mathrm{He}^{1}$ and Limin Yang ${ }^{1,4,5^{*}} \mathbb{E}$
}

\begin{abstract}
The topographic projection of afferent terminals into two-dimensional maps is essential for sensory systems to encode the locations of sensory stimuli. In vertebrates, guidance cues are critical for establishing a coarse topographic map, while neuronal activity directs fine-scale topography between adjacent afferent terminals. However, the molecular mechanism underlying activity-dependent fine-scale topography is not well known. Studies in the Drosophila visual system have demonstrated that cell-adhesion molecules direct fine-scale topography, but whether or not these molecules are involved in activity-dependent fine-scale topography remains to be determined. We previously reported that the nociceptors in Drosophila larvae form an activity-dependent fine-scale topographic system. The establishment of this system is instructed by the level of neuronal activity in individual nociceptors. Here, we show that the atypical cadherin Flamingo (Fmi) is required for establishing the nociceptor topographic map. We found that the topographic defect caused by loss of fmi was epistatic to the inhibition of neuronal activity and the overexpression of the activity-regulated gene Trim9. These results suggest that Fmi and neuronal activity interact to regulate fine-scale topography. This study provides a link between neuronal activity and the cell-adhesion molecule in the establishment of fine-scale topography.
\end{abstract}

Keywords: Fine-scale topography, Flamingo, Neuronal activity, Competence, Drosophila, Cell-adhesion molecule

\section{Introduction}

Adjacent somatosensory neurons of the same kind form fine projection maps-termed fine-scale topographic maps in the central nervous system (CNS) [1]. In vertebrates, the guidance cues are critical for establishing a coarse topographic map, and neuronal activity then directs fine-scale topography between adjacent afferent terminals [2]. In the visual system of Drosophila, many studies have shown that cell-adhesion molecules play a vital role in the formation of fine-scale topography [36]. By using genetic mosaic techniques, such as flip-out and mosaic analysis with a repressible cell marker (MARCM) [7], it was found that the cell-adhesion molecules Flamingo (Fmi) and N-cadherin (Ncad) are responsible for axon-axon interactions, which in turn

\footnotetext{
* Correspondence: yanglimin@dlu.edu.cn

${ }^{\dagger}$ Ruonan Li and Yuhua Liang contributed equally to this work.

'School of Medicine, Dalian University, Dalian 116622, Liaoning, China

${ }^{4}$ Chronic Disease Research Center, Dalian Key Laboratory, Dalian 116622,

Liaoning, China

Full list of author information is available at the end of the article
}

regulate the formation of fine-scale topography [3, 4]. The functions of Fmi and Ncad are different in establishing the topography of photoreceptor axons $[5,6]$. Fmi is mainly expressed in the photoreceptors, while Ncad is expressed in both photoreceptors and their targets $[3,4]$. Fmi mediates opposing interactions between photoreceptors, while Ncad mediates attractive interactions between photoreceptors and their targets $[5,8]$. Whether or not neuronal activity and cell-adhesion molecules interact to establish fine-scale topography is unknown.

The formation of fine-scale topography in the Drosophila visual system is independent of neural activity [9], precluding the use of this system for testing the interaction between neuronal activity and cell-adhesion molecules. We previously found that the axon terminals of Drosophila larvae nociceptors could form an activity-dependent fine-scale topographic map in ventral never cord (VNC) [10]. Thus, this system offers the opportunity for studying the interaction between 
neuronal activity and cell-adhesion molecules in the formation of fine-scale topography. In Drosophila lar$v a e$, the dendrites of the three nociceptors-- termed Class IV dendritic arborization (C4da) neurons --in each hemi-segment partition the body wall of the hemisegment. We previously found that the axon terminals of the three C4da neurons, ddaC (dorsal, D), v'ada (middle, M) and vdaB (ventral, V), form a fine-scale topographic map in the ventral nerve cord (VNC), which is Drosophila's equivalent of the vertebrate spinal cord [10]. The positions of the axon terminals in the VNC correspond to the dendritic coverages on the body wall, such that the axon of the $\mathrm{D}$ neuron projects to the dorsal side of the C4da neuropil in the VNC, the $\mathrm{M}$ neuron projects to the middle, and the $\mathrm{V}$ neuron projects to the ventral side. Moreover, we showed that the topographic separation of axon terminals between the $\mathrm{M}$ and $\mathrm{V}$ neurons depends on neuronal activity. Specifically, inhibition of neuronal activity mainly affects $M$ axon terminals and increases activity primarily affects $\mathrm{V}$ axon terminals. Neuronal activity regulates the expression of the cytoplasmic protein Tripartite motif protein, Anomalies in sensory axon patterning (Asap), which is also called Drosophila Trim9 (dTrim9). $d$ Trim9 expression level is higher in $\mathrm{V}$ neurons than in $M$ neurons [11]. Inhibition of neuronal excitability increases $d$ Trim 9 levels in $M$ neurons, while increased excitability reduces $d$ Trim9 levels in V neurons [10].

In this study, we used the C4da topographic system to test the potential contribution of Fmi to activitydependent fine-scale topography. We found that the instructive role of neuronal activity and $d$ Trim 9 in the formation of C4da topographic map disappear upon the loss of $f m i$, suggesting that Fmi determines the competence of neurons for activity-dependent fine-scale topography. This study provides mechanistic insights into the molecular mechanism underlying activity-dependent fine-scale topography.

\section{Materials and methods \\ Fly stocks}

The following published fly strains were used: $f m i^{E 59}$ [12], UAS-Fmi [12, 13], $\operatorname{asap}^{91}$ (a null allele of $d$ Trim9) [11], UAS-3HA-asap [11], UAS-kir2.1 [14, 15], UAS-ork ${ }^{\Delta N C}$, UAS-ork $^{\Delta \mathrm{C}}$ [15]. Flies are reared on standard cornmealbased food at $25^{\circ} \mathrm{C}$.

\section{Mosaic analysis for topography}

MARCM experiments were employed as previously described [7, 10]. Briefly, we used a C4da specific GAL4 driver, ppk-GAL4 [16], to express mCD8-GFP in single C4da neurons through MARCM. A transgene expressing the red fluorescent protein tdTomato directly driven by the C4da-specific $p p k$ promoter (ppk-
tdTomato) [16] is integrated into the MARCM system. The synaptic terminals of single C4da neurons were thus identified within the C4da neuropils. Three hours after egg-laying, the eggs were heat-shocked at $37.5^{\circ} \mathrm{C}$ for $15 \sim 25 \mathrm{~min}$. The eggs were kept at $25^{\circ} \mathrm{C}$ for $72 \mathrm{~h}$ after heat shock, and then dissected for immunostaining. Only earlier third instar larvae were dissected to ensure the consistency of the developmental stages of the analyzed animals.

\section{Immunohistochemistry}

Earlier third instar larval fillets were stained with the following primary antibodies: chicken anti-GFP (1:5000, Abcam), mouse anti-GFP (1:5000, Life Technologies), and rabbit anti-RFP (1:5000, Life Technologies). The following secondary antibodies were used: donkey antichicken Alexa Fluro ${ }^{\circ}$ 488(1:500, Jackson ImmunoResearch Inc), donkey anti-mouse Alexa Fluro ${ }^{\circ} 488$ (1: 500, Jackson ImmunoResearch Inc) and donkey anti-rabbit Rhodamine RX (1:500, Jackson ImmunoResearch Inc).

\section{Image preprocessing and analysis}

The image preprocessing was performed as described [10]. All images were collected as 3D stacks using an FV1000 confocal system (Olympus Microsystems) equipped with a $60 \times$ oil lens (Plan-Apochromat, numerical aperture $[\mathrm{NA}]=1.4$, Olympus Microsystems). The z-step was $0.3 \mu \mathrm{m}$. Minimum signal saturation when collecting all images and all image stacks were deconvolved with Huygens software (Scientific Volum Imaging). The 3D image analysis software Amira (FEI Visualization Sciences Group) was employed to align all of the VNC in each stack to uniform orientation. The 3D image stacks contain only the C4da neuropil with the single MARCM clones were cropped by software ImageJ (National Institutes of Health). After the above preprocessing, the image stacks were analyzed by custom-designed software for the topographic index (TI) [10]. The software measures the relative position of each clone between the dorsal and ventral boundaries of a C4da neuropil and calculates the TI of each clone. The final TI for each clone was then calculated as the average of $\mathrm{TI}=\Sigma \mathrm{TI} i / \mathrm{n}$, where $\mathrm{n}$ is the total number of cloned voxels in the 3D image stack. The TI analysis was done by the software automatically.

\section{Statistical analysis}

All data are presented as mean values \pm SEM. The data were analyzed with Prism GraphPad 6.01. The student's $t$-test performed a data comparison between the two groups. One-way ANOVA with Sidák correction was 
used for comparing three or more groups. $P<0.05$ was considered significant.

\section{Results \\ Flamingo is required for establishing the fine-scale topography of C4da neurons}

The axon terminals of the three nociceptors in each larval hemisegment form an expanded structure in the VNC that is enriched with synapses, which is termed the C4da neuropil [17]. The axon terminals of D, M, and V nociceptors are positioned in the dorsal, middle, and ventral parts of the C4da neuropil, forming a finetopographic map [10]. The C4da topographic map is established gradually during the development. First, at the embryonic stage, the D axon was separated from the $\mathrm{M}$ and $\mathrm{V}$ axons by projecting dorsally and entered the C4da neuropil through the dorsal bundle. Second, the terminals of $\mathrm{M}$ and $\mathrm{V}$ neurons are separated at the early second-instar stage through a neuronal-activitydependent process (Fig. 1a).

Prompted by previous findings that the cell adhesion molecule Fmi regulates fine-scale topography in Drosophila visual system by mediates axon-axon interaction, we set out to determine whether Fmi plays a role in the fine-scale topography in the C4da system. Using the single-cell genetic manipulation technique MARCM, we found that loss of $f m i$ in single $\mathrm{V}$ neurons shifted the presynaptic terminals of these neurons dorsally to the middle portion of the C4da neuropil. The positions of presynaptic terminals of fmi-/- D and $\mathrm{M}$ neurons remained indistinguishable from those of wild-type $\mathrm{D}$ and $\mathrm{M}$ neurons (Fig. 1b, c). Overexpression of Fmi in single C4da neurons did not affect the topographic map (Fig. 1b, c), but completely rescued the topographic defect in fmi-/$\mathrm{V}$ neurons (Fig. 1d). These results suggested that the topographic arrangement of the $\mathrm{M}$ and $\mathrm{V}$ terminals requires $f m i$.

In addition to the topographic defect, loss of fmi also reduced the length of $\mathrm{C} 4 \mathrm{da}$ axon terminals, whereas overexpression of Fmi led to an overgrowth of these terminals (Fig. 2). These results suggest that Fmi instructs the growth of C4da presynaptic terminals. Fmi's function in presynaptic growth seems to be distinct from that in topography because the only loss of fmi, but not overexpression, affected the C4da topography.

\section{Flamingo determines the competence of C4da neurons for Trim9-instructed topography}

The $d$ Trim 9 instructs the establishment of C4da topography [10]. The topographic defect caused by loss of $f m i$ in single C4da neurons was similar to that caused by loss of $d \operatorname{Trim} 9$ and opposite to $d \operatorname{Trim} 9$ overexpression. Thus, we decided to test whether $d \operatorname{Trim} 9$ and fmi genetically interact with each other to determine fine-scale topography. Epistasis tests were performed to determine whether the topographic effects of $d$ Trim9 requires Fmi function. Using the MARCM technique, we overexpressed $d$ Trim 9 in either wild-type or fmi-/- C4da neurons. Wild-type M neurons overexpressing $d$ Trim 9 projected their presynaptic terminals to the ventral portion of the C4da neuropil [10] (Fig. 3a, b). This topographic defect was entirely blocked by the loss of fmi (Fig. 3a, b). By contrast, overexpressing $\mathrm{Fmi}$ in $d$ Trim9-/- neurons did not change the topographic phenotype of $d \operatorname{Trim} 9$ $-/-$, which is a dorsal shift of the presynaptic terminals of $\mathrm{V}$ neurons (Fig. 3c, d). These results suggested that the capability of $d \mathrm{Trim} 9$ in regulating C4da topography requires Fmi. In other words, Fmi confers C4da neurons the competence for $d$ Trim9-instructed topography.

In contrast to the genetic interactions in topography, overexpressing Fmi in $d$ Trim9-/- neurons partially rescued the length and branch numbers of axon terminals (Fig. 2). Moreover, while fmi-/- completely eliminated of the topographic defects caused by $d$ Trim 9 overexpression, it only partially blocked the axon morphological defects caused by $d$ Trim 9 overexpression (Fig. 2). These results again suggest that the morphology and fine-scale topography of C4da axon terminals are regulated by different mechanisms.

\section{Flamingo determines the competence of C4da neurons for activity-dependent topography}

The separation of the presynaptic terminals of $M$ and $\mathrm{V}$ neurons is activity-dependent. Neural activities of $\mathrm{M}$ and $\mathrm{V}$ neurons regulate $d$ Trim 9 levels to control the topographic positions of the $\mathrm{M}$ and $\mathrm{V}$ neurons [10]. The inhibition of neuronal activity results in a topographic defect that is similar to $d$ Trim 9 overexpression (Fig. 1a). Since $d$ Trim 9 requires Fmi in instructing topographic projection, we asked whether neuronal activities of $\mathrm{C} 4 \mathrm{da}$ neurons also require $\mathrm{Fmi}$ to instruct $\mathrm{C} 4 \mathrm{da}$ topography. Because inhibiting neuronal activity resulted in a topographic defect that was opposite to fmi loss of function, we performed a genetic epistasis test to detect whether the neuronal activity requires $f m i$ to regulate C4da topography. Using the MARCM technique, we overexpressed the inward rectifier potassium channel Kir2.1 [18] in fmi-/C4da neurons to robustly inhibit the activity of them. The presynaptic terminals of $f m i-/-M$ neurons that overexpressed Kir2.1 shifted dorsally to the middle position (Fig. 4a, c), demonstrating that the topographic phenotype caused by Kir2.1 overexpression requires fmi. Replacing Kir2.1 with a constitutively 

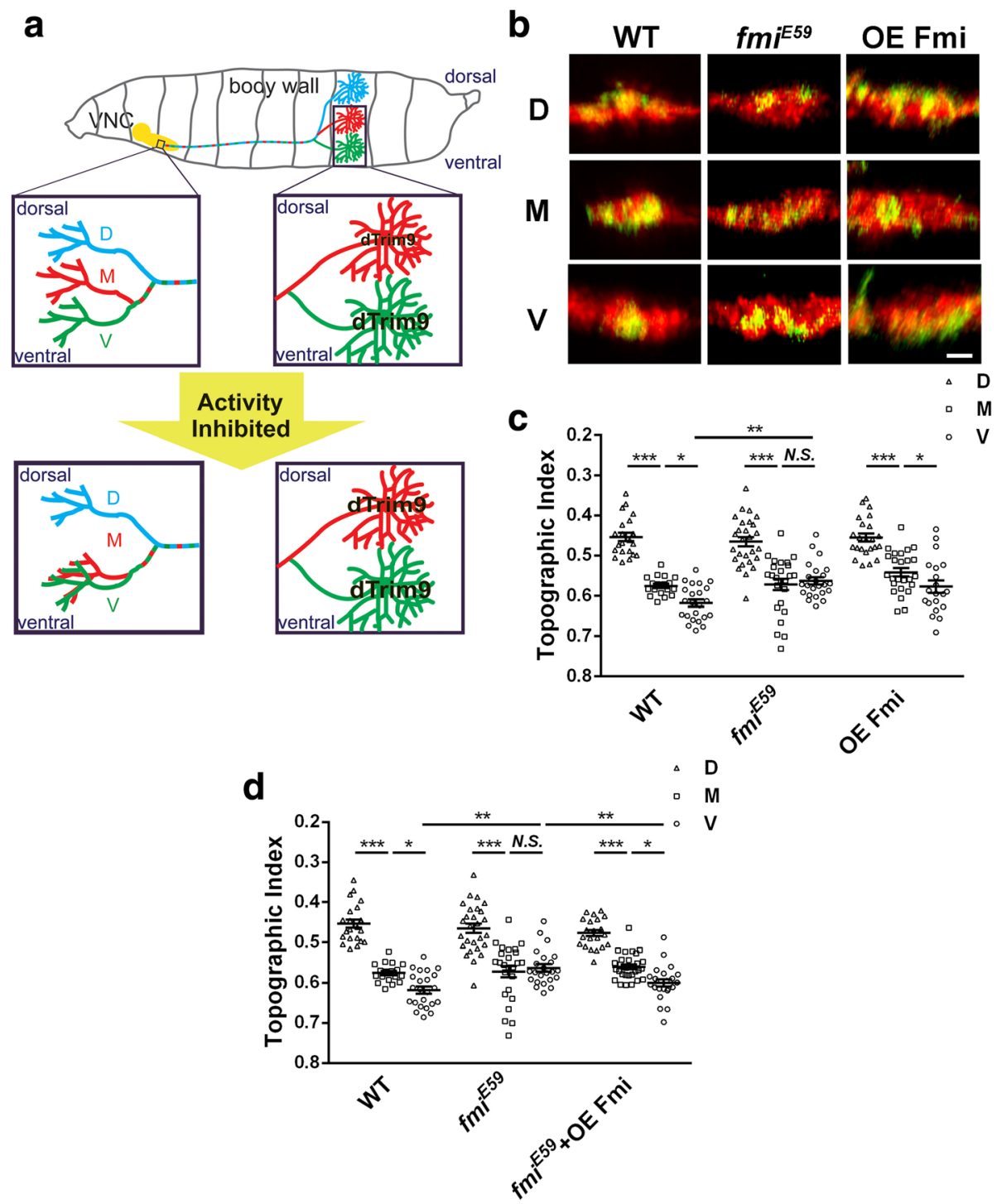

Fig. 1 Flamingo is required for the formation of the C4da topographic map. a The three $C 4 d a$ neurons $(D, M$ and $V$ ) in one hemi-segment of Drosophila larvae project into VNC to form an activity-dependent topographic map. This topography depends on the relative levels of $d$ Tim9 in these three neurons. The activity inhibition of $\mathrm{M}$ neurons shifts their axon terminals ventrally by increasing $d$ Trim 9 expression. $\mathbf{b}$ Representative images of the dorsal-ventral view of single C4da terminals (green) and the C4da neuropil marked by ppk-tdTomato (red). WT, wild type; fmi ${ }^{E 59}$, fmi null allele of mutation; OE Fmi, overexpress flamingo. c The statistic analysis of the TI of C4da axon terminals. Fmi null mutation causes the axon terminals of $\mathrm{V}$ neurons to project toward the dorsal side of $\mathrm{VNC}$, resulting in the mixing of $\mathrm{M}$ terminals with $\mathrm{V}$ terminals. There is a significant difference in the projection of the V-terminals of the fmi mutation and the wild type V-terminals in VNC. Overexpress Fmi has no effects on the formation of topography. $\mathbf{d}$ Overexpress Fmi rescues the topographic defect in fmi mutant C4da neurons. The axon terminals of fmi mutatnt $V$ neurons relocated to the ventral side of C4da neuropil by overexpressing Fmi on it. There is a significant difference in the projection of $\mathrm{V}$ terminals of the fmi mutation and the fmi mutation + OE Fmi. D, ddaC; $\mathrm{M}$, v'ada; $\mathrm{V}$, vdaB. ${ }^{*} P<0.05 ;{ }^{* *} P<0.01 ;{ }^{* *} P<0.001$; Scale bar, $2 \mu \mathrm{m}$. Error bars indicate mean \pm SEM. WT: $\mathrm{D}, n=22 ; \mathrm{M}, n=18 ; \mathrm{V}, n=23 ; \mathrm{fmi}^{\mathrm{E} 59}$ : $\mathrm{D}, n=27 ; \mathrm{M}, n=25 ; \mathrm{V}, \mathrm{n}=23 ; \mathrm{OE}$ Fmi: $\mathrm{D}, \mathrm{n}=23 ; \mathrm{M}, \mathrm{n}=25 ; \mathrm{V}, n=20$; $\mathrm{fmi}^{\mathrm{E} 59}+\mathrm{OE}$ Fmi: $\mathrm{D}, n=21 ; \mathrm{M}, n=29 ; \mathrm{V}, \mathrm{n}=23$

open mutant of Drosophila rectifier potassium channel $1\left(d \mathrm{ORK}^{\Delta \mathrm{C}}\right)$ [18] had similar effects (Fig. 4b, d), confirming the results of Kir2.1 overexpression.

Taken together, these results show that, just like $d$ Trim9 overexpression, the effect of inhibiting neuronal activity on C4da topography requires Fmi. They suggest that Fmi determines the competence of $\mathrm{M}$ and $\mathrm{V}$ terminals for topographic separation. Unlike overexpression of $d$ Trim9, overexpressing Kir2.1 reduced the number of axon terminal branches in fmi mutant neurons (Fig. 4f), but did not affect the length of axon terminals (Fig. 4e). This may suggest that neuronal activity and $d$ Trim 9 


\section{a}

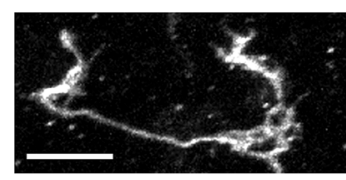

WT

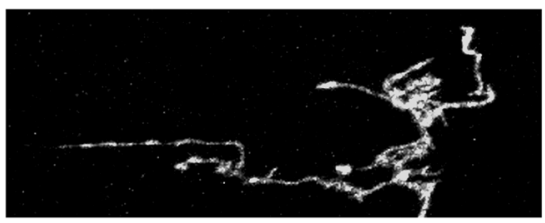

OE Fmi

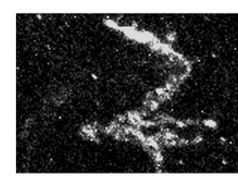

fmi ${ }^{\mathbf{E} 59}$

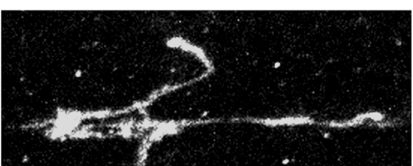

fmi ${ }^{\mathrm{F} 59}+\mathrm{OE}$ dTrim9

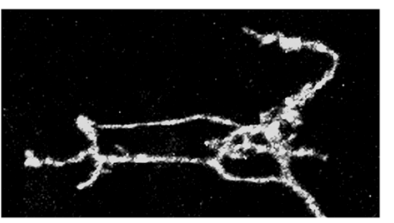

OE Fmi+dTrim9-/- b

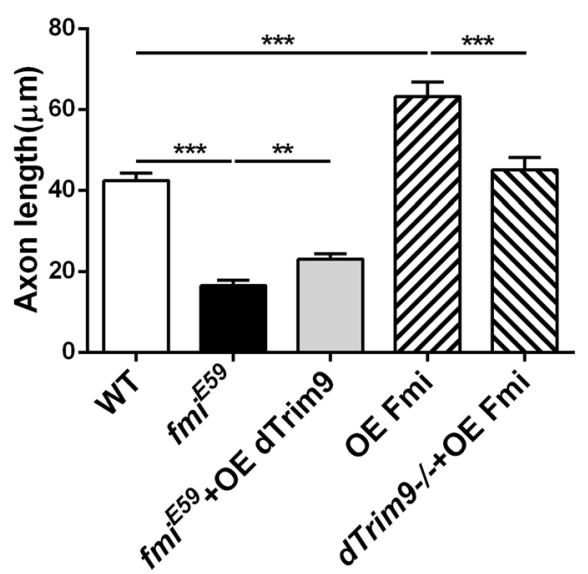

C

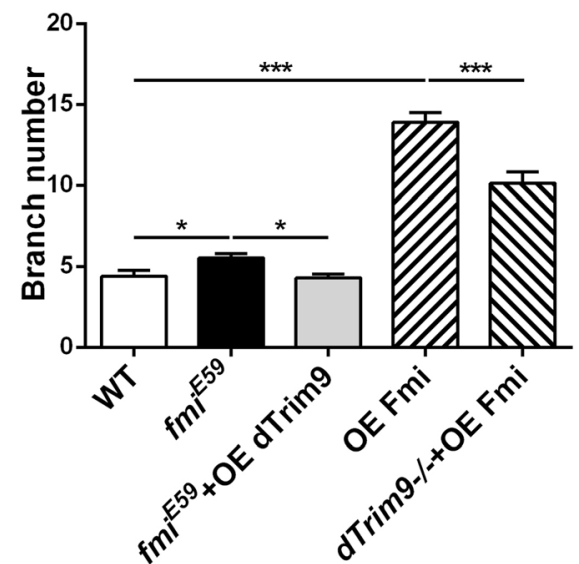

Fig. 2 Effects of Fmi on the growth of C4da axon terminals. a Images of $V$ axon terminals. $\mathbf{b}$, $\mathbf{c}$ fmi mutations lead to shortening of axon terminals and an increase in the number of branches of $V$ neurons. Overexpression of Fmi leads to excessive growth of axon terminals and an increase in the number of branches. dTrim9 partially rescues the phenotypes caused by fmi dysfunction. ${ }^{*} \mathrm{P}<0.05 ;{ }^{*} \mathrm{P}<0.01$; ${ }^{* *} \mathrm{P}<0.001 ; \mathrm{WT}$ : $\mathrm{n}=$ 22; fmi ${ }^{E 59}: \mathrm{n}=20 ; f_{m i}{ }^{E 59}+$ OE dTrim9: $n=19 ;$ OE Fmi: $\mathrm{n}=20$; dTrim9-1- + OE Fmi: $\mathrm{n}=18$

regulate axon terminal morphology through different pathways.

\section{Discussion}

In this study, we examined the role of Fmi in the formation of C4da fine-scale topographic map in Drosophila larvae. We found that Fmi is required for the neuronal activity and $d$ Trim 9 to regulate the formation of fine-scale topography. We also show an instructive role of $\mathrm{Fmi}$ in the growth of C4da axon terminals, which appears to be a distinct function from topography.

By using single-cell gene manipulation technique MARCM, we found that loss of fmi function leads to the disappearance of the topographic separation of the presynaptic terminals of $\mathrm{M}$ neurons and $\mathrm{V}$ neurons by shifting $\mathrm{V}$ terminals dorsally. Overexpression of Fmi did not affect the C4da topography (Fig. 1c), suggesting that Fmi plays a permissive, but not an instructive role, in establishing C4da topography. This is different from the mechanism of action of Fmi in the Drosophila visual system. In the Drosophila visual system, both loss and overexpression of Fmi can lead to defects in photoreceptor R1-R6 target selection, and the effect is nonautonomous [5]. Although we did not observe the phenotype of wild-type C4da axon terminals that are surrounded by fmi-/- C4da neurons, we speculate that Fmi's function in C4da neurons is cell-autonomous, because the MARCM experiments showed that fmi-/lead defects of $\mathrm{M}$ and $\mathrm{V}$ projections. This suggests that the mechanism of action of Fmi in the visual system is different from that in the C4da neurons.

We previously showed that C4da topography is activity-dependent and that neuronal activity determines the expression of $d$ Trim 9 protein to instruct the formation of C4da fine-scale topography [10] (Fig. 
a

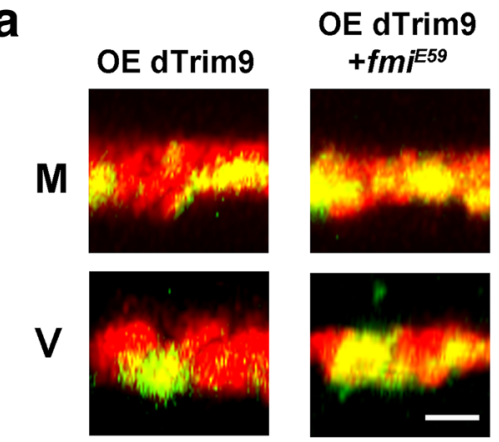

C

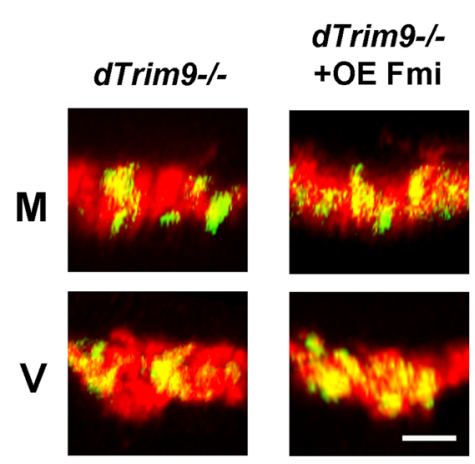

\section{b}
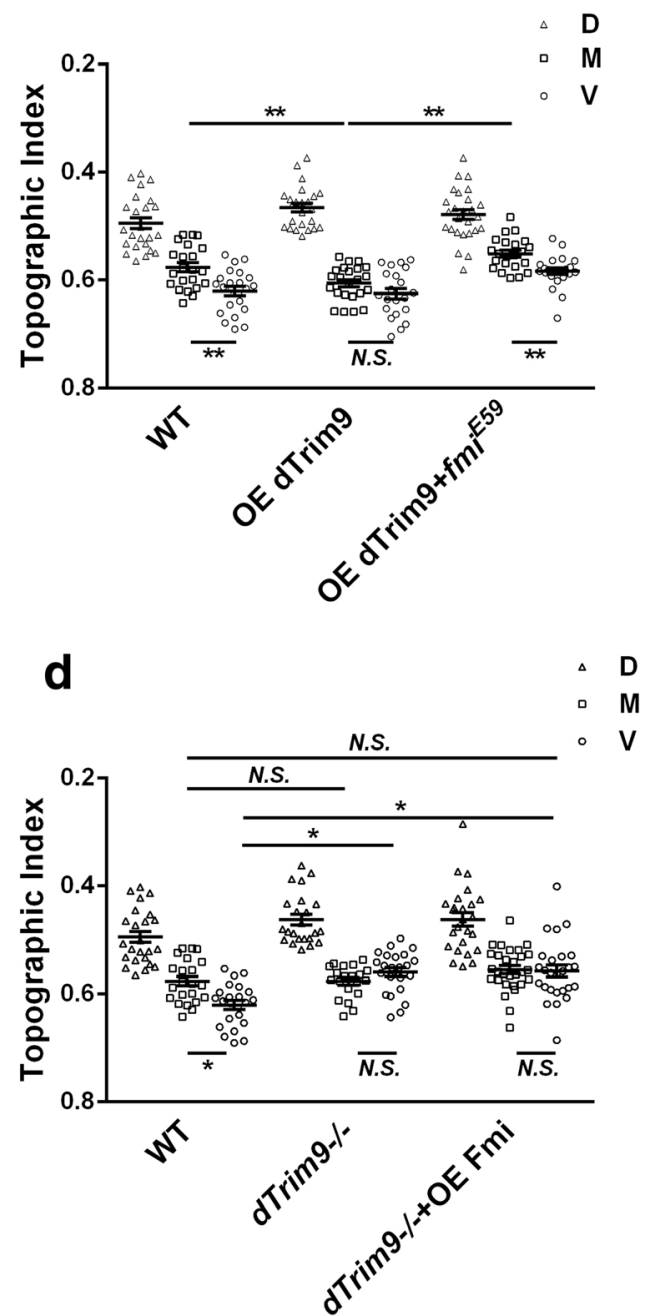

Fig. 3 Genetic interactions between Fmi and dTrim9 in C4da topography. a, c Representative images of the dorsal-ventral view of single C4da terminals (green) and the C4da neuropil marked by ppk-tdTomato (red). $\mathbf{b}$ Overexpression of dTrim9 causes the axon terminals of $\mathrm{M}$ neurons to shift to the ventral side, mixing with the axon terminals of $V$ neurons, resulting in a defect in topography, but this phenotype disappears in the fmi mutant. c The statistic results of axon terminals TI of three neurons of C4da. Overexpression of Fmi has no effects on the phenotype of the dTrim9 mutation. OE, overexpress; $-/-$, null allele of mutation; $\mathrm{D}, \mathrm{ddaC} ; \mathrm{M}, \mathrm{v}$ 'ada; $\mathrm{V}$, vdaB. ${ }^{*} P<0.05 ;{ }^{*} P<0.01 ;$ N.S., no significant difference. Scale bar, $2 \mu \mathrm{m}$. Error bars indicate mean \pm SEM. WT: $D, n=24 ; M, n=22 ; V, n=23 ; \mathrm{OE} d \operatorname{Trim9}: \mathrm{D}, \mathrm{n}=24 ; \mathrm{M}, \mathrm{n}=23 ; \mathrm{V}, \mathrm{n}=21 ; \mathrm{OE} d \operatorname{Trim} 9+$ fmi ${ }^{E 59}: \mathrm{D}$, $n=26 ; M, n=22 ; V, n=23 ; d T r i m 9-/-: D, n=23 ; M, n=20 ; V, n=23 ; d \operatorname{Trim} 9-/-+$ OE Fmi: $D, n=25 ; M, n=29 ; V, n=26$

1a). It is different from the formation of visual finescale topography, which is independent of the neural activity [9]. Whether it is activity-dependent or nonactivity-dependent fine-scale topography, their formations are closely related to the establishment and maintenance of synapses between pre- and postsynaptic neurons. Studies have shown that neurons require the cell adhesion molecule Fmi for synaptic target selection, synaptogenesis, the survival of axons and synapses $[13,19]$. We speculate that in the formation of C4da fine-scale topography, the activity- $d$ Trim9 signaling pathway instructs the axon of $\mathrm{M}$ and $\mathrm{V}$ neurons to the target area in C4da neuropil, and then
Fmi helps to form synapse between these neurons and their postsynaptic targets. When Fmi is absent, C4da axon terminals fail to stabilize in the target area and do not form correct synapses. Bao et al. showed that $f m i$ mutation causes a significant increase in the number of ectopic synapses on muscles [13]. In the C4da system, loss of fmi may cause the axon terminals of $\mathrm{V}$ neuron to form ectopic synapses, resulting in a dorsal shift of these axon terminals to the middle of C4da neuropil. Supporting this idea, we found that loss of fmi led to an increase in the C4da axon terminal branch number, which may reflect ectopic synapse formation. Overexpression of $d$ Trim 9 and 
a
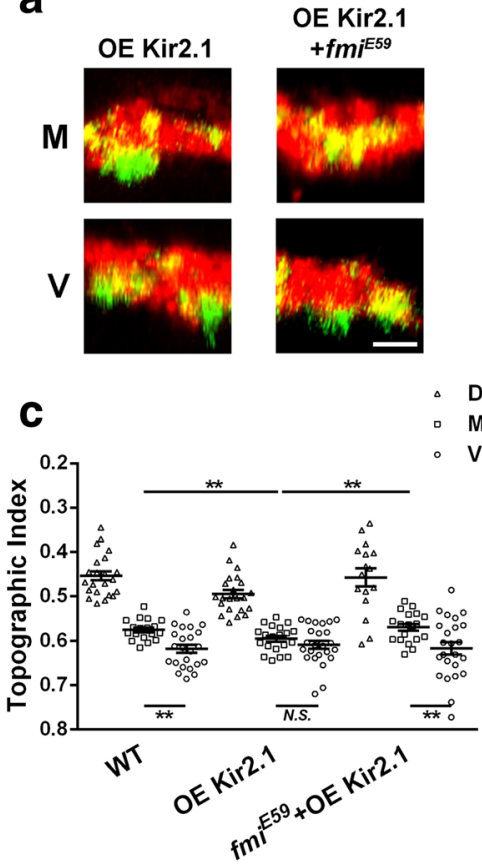

e
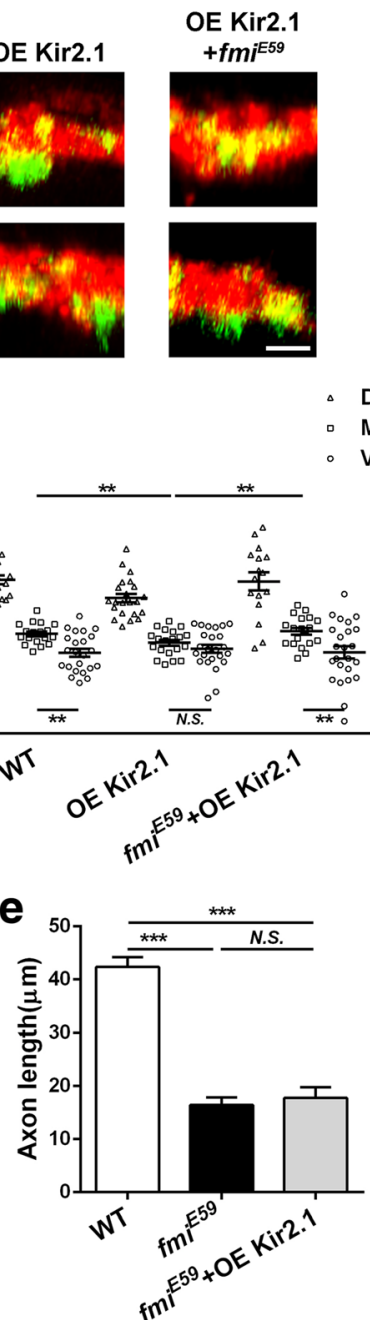

b
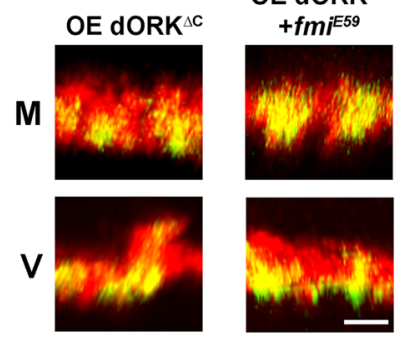

OE dORK $\triangle \mathrm{C}$

$+f m i=59$

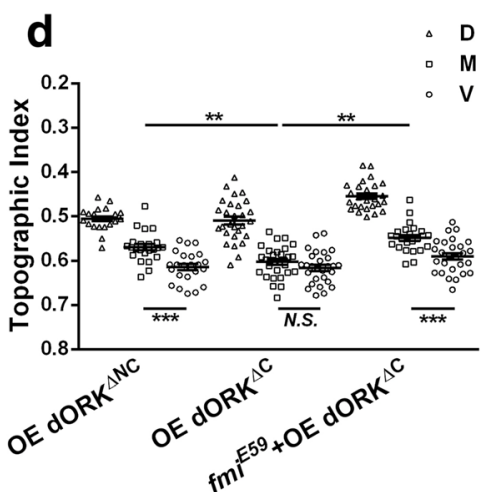

\section{f}

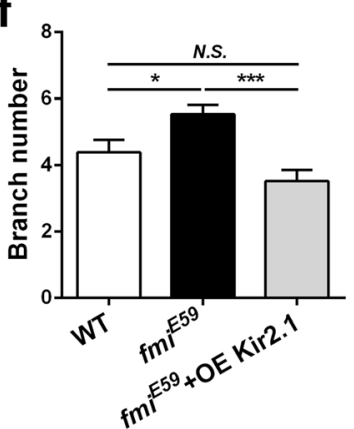

Fig. 4 Neuronal activity requires Fmi to instruct C4da topography. a, b Representative images of the dorsal-ventral view of single C4da terminals (green) and the C4da neuropil marked by ppk-tdTomato (red). $\mathbf{c}, \mathbf{d}$ are statistic results of the axon terminals index of three neurons of C4da. a, c Inhibition of neuronal activity by overexpressing Kir2.1. $\mathbf{b}$, d Inhibition of the neuronal activity of C4da neurons by overexpressing ORK ${ }^{\triangle N C}$ as $W T$ and $\mathrm{ORK}^{\triangle \mathrm{C}}$. Both $\mathbf{c}$, d showed that in the fmi mutant neurons, the regulation of neuronal activity on topography disappeared. $\mathbf{c} W T: D, n=22 ; M, n=18 ; V, n=23 ; O E$ Kir2.1: $D, n=23 ; M, n=20 ; V, n=24 ; f m i^{E 59}+O E$ Kir2.1: $D, n=16 ; M$, $n=19 ; V, n=24$. d WT: $D, n=22 ; M, n=23 ; V, n=23 ;$ OE ORK ${ }^{\Delta C}: D, n=28 ; M, n=30 ; V, n=28 ; f m i i^{559}+O_{E} O R K^{\Delta C}: D, n=26 ; M, n=24 ;$ $\mathrm{V}, n=27$. e Inhibition of neuronal activity does not affect the axonal shortening caused by loss of fmi. $\mathbf{f}$ Inhibition of neuronal activity can rescue the increased branch's number of axon terminals caused by fmi mutations. $\mathrm{D}$, ddaC; $\mathrm{M}, \mathrm{v}$ 'ada; $\mathrm{V}, \mathrm{vdaB}$. ${ }^{*} \mathrm{P}<0.05 ;{ }^{*} \mathrm{P}<0.01$; ${ }^{* * *} P<0.001 ;$ N.S., no significant difference. Scale bar, $2 \mu \mathrm{m}$. Error bars indicate mean \pm SEM. WT: $\mathrm{n}=22 ; \mathrm{OE}$ Kir2.1: $\mathrm{n}=20 ; f m i i^{E 59}+\mathrm{OE}$ Kir2.1: $n=17$

inhibition of neuronal activity reduced the increased branch number caused by fmi mutations (Figs. 2c and 4f). To verify this hypothesis, further experiments are needed to observe the changes in synapse distribution of the axon terminals in C4da neurons directly, which is technically challenging at this stage.

In summary, neuronal activity plays an instructive role in the formation of C4da topography, but this role requires the presence of Fmi. Fmi determines the competence of neurons for activity-dependent fine-scale topography.

\section{Abbreviations}

C4da neurons: Class IV dendritic arborization neurons; CNS: central nervous system; Fmi: Flamingo; MARCM: mosaic analysis with a repressible cell marker; Ncad: N-cadherin; TI: topographic index; VNC: ventral never cord

\section{Acknowledgments}

We thank Dr. Bing Ye for sharing the fly stocks, and Dr. Takuya Kaneko for assistance in image deconvolution.

\section{Authors' contributions}

LMY designed, performed experiments, analyzed data, and drafted the manuscript. RNL and YHL performed MARCM experiments, analyzed data, and contributed to the writing of the manuscript. SYZ and QH performed 
eggs collection and data analysis. All authors read and approved the final manuscript.

\section{Funding}

This study was funded by the National Natural Science Foundation of China (Grant No. 31571087 and 31200839).

\section{Availability of data and materials}

The datasets generated and/or analyzed during the current study are available from the corresponding author on reasonable request.

\section{Ethics approval}

Not applicable.

\section{Consent for publication}

Not applicable.

\section{Competing interests}

The authors declare that they have no competing interests.

\section{Author details}

${ }^{1}$ School of Medicine, Dalian University, Dalian 116622, Liaoning, China. ${ }^{2}$ Institute of Health Sciences, China Medical University, Shenyang 110112, China. ${ }^{3}$ Life Sciences Institute, Dalian National University, Dalian 116600 , Liaoning, China. ${ }^{4}$ Chronic Disease Research Center, Dalian Key Laboratory, Dalian 116622, Liaoning, China. ${ }^{5}$ School of Medicine, Jiamusi University, Jiamusi 154007, Heilongjiang, China.

Received: 21 September 2019 Accepted: 28 November 2019 Published online: 10 December 2019

\section{References}

1. Katz LC, Shatz CJ. Synaptic activity and the construction of cortical circuits. Science. 1996;274(5290):1133-8.

2. Pfeiffenberger C, Yamada J, Feldheim DA. Ephrin-As and patterned retinal activity act together in the development of topographic maps in the primary visual system. J Neurosci. 2006;26(50):12873-84.

3. Lee $\mathrm{CH}$, Herman T, Clandinin TR, Lee R, Zipursky SL. N-cadherin regulates target specificity in the Drosophila visual system. Neuron. 2001;30(2):437-50

4. Lee RC, Clandinin TR, Lee CH, Chen PL, Meinertzhagen IA, Zipursky SL. The protocadherin flamingo is required for axon target selection in the Drosophila visual system. Nat Neurosci. 2003;6(6):557-63.

5. Chen PL, Clandinin TR. The cadherin flamingo mediates level-dependent interactions that guide photoreceptor target choice in Drosophila. Neuron. 2008;58(1):26-33.

6. Schwabe T, Neuert $\mathrm{H}$, Clandinin TR. A network of cadherin-mediated interactions polarizes growth cones to determine targeting specificity. Cell. 2013:154(2):351-64.

7. Lee T, Luo L. Mosaic analysis with a repressible cell marker for studies of gene function in neuronal morphogenesis. Neuron. 1999;22(3):451-61.

8. Prakash S, Caldwell JC, Eberl DF, Clandinin TR. Drosophila N-cadherin mediates an attractive interaction between photoreceptor axons and their targets. Nat Neurosci. 2005;8(4):443-50.

9. Hiesinger PR, Zhai RG, Zhou Y, Koh TW, Mehta SQ, Schulze KL, Cao Y, Verstreken $\mathrm{P}$, Clandinin TR, Fischbach KF, et al. Activity-independent prespecification of synaptic partners in the visual map of Drosophila. Curr Biol. 2006;16(18):1835-43.

10. Yang L, Li R, Kaneko T, Takle K, Morikawa RK, Essex L, Wang X, Zhou J, Emoto K, Xiang Y, et al. Trim9 regulates activity-dependent fine-scale topography in Drosophila. Curr Biol. 2014;24(9):1024-30.

11. Morikawa RK, Kanamori T, Yasunaga K, Emoto K. Different levels of the tripartite motif protein, Anomalies in sensory axon patterning (Asap), regulate distinct axonal projections of Drosophila sensory neurons. Proc Natl Acad Sci U S A. 2011:108(48):19389-94.

12. Usui T, Shima Y, Shimada Y, Hirano S, Burgess RW, Schwarz TL, Takeichi M, Uemura T. Flamingo, a seven-pass transmembrane cadherin, regulates planar cell polarity under the control of frizzled. Cell. 1999;98(5):585-95.

13. Bao H, Berlanga ML, Xue M, Hapip SM, Daniels RW, Mendenhall JM, Alcantara AA, Zhang B. The atypical cadherin flamingo regulates synaptogenesis and helps prevent axonal and synaptic degeneration in Drosophila. Mol Cell Neurosci. 2007;34(4):662-78.
14. Baines RA, Uhler JP, Thompson A, Sweeney ST, Bate M. Altered electrical properties in Drosophila neurons developing without synaptic transmission. J Neurosci. 2001;21(5):1523-31.

15. Nitabach MN, Blau J, Holmes TC. Electrical silencing of Drosophila pacemaker neurons stops the free-running circadian clock. Cell. 2002;109(4):485-95.

16. Grueber WB, Ye B, Moore AW, Jan LY, Jan YN. Dendrites of distinct classes of Drosophila sensory neurons show different capacities for homotypic repulsion. Curr Biol. 2003;13(8):618-26.

17. Zlatic M, Li F, Strigini M, Grueber W, Bate M. Positional cues in the Drosophila nerve cord: semaphorins pattern the dorso-ventral axis. PLoS Biol. 2009:7(6):e1000135.

18. Nitabach MN, Llamas DA, Thompson IJ, Collins KA, Holmes TC. Phosphorylation-dependent and phosphorylation-independent modes of modulation of shaker family voltage-gated potassium channels by SRC family protein tyrosine kinases. J Neurosci. 2002:22(18):7913-22.

19. Hakeda-Suzuki S, Berger-Muller S, Tomasi T, Usui T, Horiuchi SY, Uemura T, Suzuki T. Golden goal collaborates with flamingo in conferring synapticlayer specificity in the visual system. Nat Neurosci. 2011;14(3):314-23.

\section{Publisher's Note}

Springer Nature remains neutral with regard to jurisdictional claims in published maps and institutional affiliations.
Ready to submit your research? Choose BMC and benefit from:

- fast, convenient online submission

- thorough peer review by experienced researchers in your field

- rapid publication on acceptance

- support for research data, including large and complex data types

- gold Open Access which fosters wider collaboration and increased citations

- maximum visibility for your research: over $100 \mathrm{M}$ website views per year

At $\mathrm{BMC}$, research is always in progress.

Learn more biomedcentral.com/submissions 